\title{
India plans to bring neutrino science home
}

\section{K. S. Jayaraman, New Delhi}

Indian high-energy physicists are seeking to revive neutrino science in their country by building an underground physics laboratory. If all goes to plan, the proposed India-based Neutrino Observatory (INO) could be operational by 2010.

Atmospheric neutrinos were first detected in 1965 by a joint Indian-British-Japanese experiment at the Kolar Gold Field in southern India. But the facility was abandoned in 1992, and the mine that housed it closed.

Now, with Europe's main neutrino lab temporarily closed and plans for an American lab in trouble, India is spending US $\$ 1$ million on a feasibility study for setting up a facility of its own, using a huge slab of magnetized iron to detect neutrinos.

"The study will be completed by the middle of next year and then submitted for funding," says Naba K. Mondal, a particle physicist at the Tata Institute of Fundamental Research in Mumbai, and INO project leader. The project would cost a minimum of 2.5 billion rupees (US\$54 million). "We are proposing an Indian-based facility but are looking for international participation," Mondal says. The INO collaboration already has 55 members from 15 Indian institutions and universities.

Two potential sites for the lab have been identified - beneath the Nilgiri Hills in the southern state of Tamilnadu, and at Rammam in West Bengal. Both are man-made caves and have more than 1,500 metres of rock cover that would shield the laboratory from cosmic radiation.

Recent environmental problems at the Gran Sasso National Laboratory in Italy (see Nature 423, 675; 2003) and delays over the US project (see Nature 423, 674; 2003) "make the idea of a new laboratory in India more attractive to the particle-physics community", says Maury Goodman, a highenergy physicist at the Argonne National Laboratory in Illinois.

Physicists are keen to improve their understanding of the properties of the three different types of neutrinos - electron, muon and tau neutrinos. Mondal says that the new laboratory could verify results obtained by physicists at Japan's SuperKamiokande detector suggesting that neutrinos can 'oscillate' between the three types. He claims that a few years of observations could confirm that neutrinos have mass and can oscillate.

This might be achieved by 'long-baseline' neutrino experiments to detect particles generated by distant accelerators in Japan, the United States or Europe. The extra distance would give neutrinos more time to oscillate between the source and the detector. "No other proposed facility has the

capabilities of this project," says Goodman.

John Learned, a neutrino physicist at the University of Hawaii, says that the plan "meets the criteria of being unique and taking advantage of local resources. It could achieve significant advances in particle physics and astrophysics."

But India may struggle to fund the facility. Final approval rests with the Department of Atomic Energy and the science ministry, whose budgets would have to support the neutrino observatory. Mondal says that funding will also be sought from the Ministry of Education, and adds: "If we undertake such a project, then we must have the involvement of Indian industry in a big way".

\section{Discovery changes view of bacteria}

\section{Jonathan Knight, San Francisco}

Bacteria have specialized compartments that were previously thought to exist only in higher organisms, according to research published earlier this month.

The discovery upsets a long-held notion about the nature of bacterial cells, researchers say. Whereas the cells of all other living things rely on a wide assortment of internal enclosures, known as organelles, to carry out their metabolic tasks, bacteria were thought to manage with a single open space devoid of partitions.

Now a team led by microbiologist Roberto Docampo at the University of Illinois in Urbana say they have found the first organelles in bacteria that have a direct counterpart in higher organisms (M. Seufferheld et al. J. Biol. Chem. doi:10.1074/jbc.M304548200; 2003). The organelles - called acidocalcisomes are thought to store energy and help control acidity within the cell.

Docampo's bacterial organelles seem to be widespread; in fact, microscopists have been looking at them for a century without

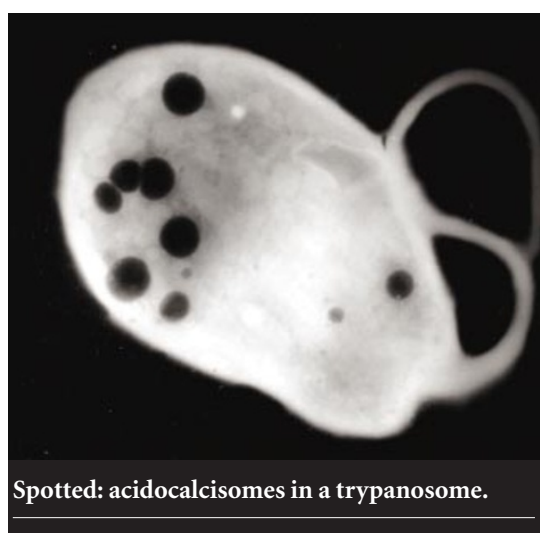

knowing it. They stain purple with certain dyes and have been called 'metachromatic' granules, but they were thought to have no membrane or enzymes - essential components of organelles.

Docampo has shown by electron microscopy and antibody techniques that metachromatic granules are surrounded by a membrane bearing proton-pumping enzymes that make its contents more acidic. For his experiments, he used Agrobacterium tumefaciens, a plant pathogen that causes crown gall. But a variety of other bacteria also have metachromatic granules, including the ulcer bug, Helicobacter pylori, and the bacterium that causes diphtheria, Corynebacterium diphtheriae.

The origin of the organelles is a mystery. In most cells, organelles fall into one of two categories. They are either components of an elaborate intracellular trafficking system, which bacteria lack, or they are derived from formerly free-living organisms that joined up with the ancient ancestors of modern cells.

Neither possibility seems particularly likely in this case, says Andrew Roger, who studies organelles at Dalhousie University in Nova Scotia and was surprised by the news. "Which of these might happen in bacteria is not clear," he says.

As one approach to this question, Docampo's group is now searching for more organisms with acidocalcisomes for comparison, by scouring sequence databases for the gene that encodes the proton pump. In addition, as the proton pump in the membrane is present in some harmful bacteria but not in human cells, drugs that target it might be effective against these infections, Docampo suggests. 